\section{Controlling CENPA mislocalization}

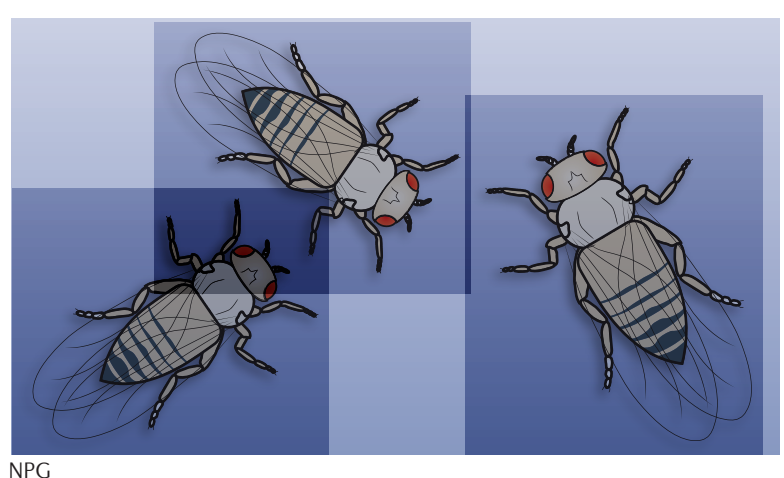

NPG

The specific deposition of the centromere-specific histone variant centromere protein A (CENPA) is key for proper kinetochore assembly and chromosome segregation. Erhardt and colleagues report that in Drosophila melanogaster, the histone-fold protein Chromatin accessibility complex $14 \mathrm{kD}$ (Chrac14)

mutating or depleting Chrac14 interacts with CENPA, regulates its causes ... ectopic kinetochore formation chromatin localization after DNA damage and prevents aneuploidy.

Chrac14 associates with several chromatin remodelling complexes and was identified by 5 the authors in a genetic screen as a candidate modulator of CENPA chromatin misincorporation. Althogh Chrac14-mutant flies seem normal, exposing their larvae to ionizing radiation causes severe developmental defects and high mortality. In tissues of irradiated Chrac14-mutant flies, damaged cells fail to activate the G2/M checkpoint and proceed to mitosis. Furthermore, in Chrac14-depleted fly S2 cells, DNA damage repair kinetics are noticeably attenuated. This indicates that Chrac14 has a role in the DNA damage response.

The depletion of Chrac14 in S2 cells also resulted in a significant increase in the number of CENPA immunofluorescence foci (also observed in Chrac14-mutant embryos), which is indicative of ectopic CENPA incorporation into chromatin, and caused ectopic kinetochore assembly, which was evident by CENPA foci colocalization with kinetochore proteins. Mitotic spreads from Chrac14-depleted cells or Chrac14-mutant embryos showed multiple CENPA foci per chromosome and, consequently, mitotic defects, such as dicentric chromosomes and anaphase bridges, lagging chromosomes and chromosomal breaks. Thus, mutating or depleting Chrac14 causes CENPA mislocalization and ectopic kinetochore formation, and results in chromosome segregation defects.

Whereas CENPA is not recruited to DNA lesions in normal cells, inducing DNA damage in Chrac14-depleted cells resulted in a significant increase in the colocalization of the phosphorylated histone variant $\mathrm{H} 2 \mathrm{Av}$ (a marker of DNA damage) and CENPA nuclear foci, which indicates that Chrac14 may prevent CENPA deposition at DNA lesions. In support of this, the authors found that both proteins interact in embryos after irradiation. Based on their findings, they propose that during DNA damage repair nucleosomes are formed using the available histone pool, including variants such as CENPA. Misincorporation of CENPA can lead to ectopic kinetochore formation, and this is prevented and/or corrected by a Chrac14-dependent mechanism. Eytan Zlotorynski

ORIGINAL RESEARCH PAPER Mathew, V. et al. The histone-fold protein CHRAC14 influences chromatin composition in response to DNA damage. Cell Rep. http://dx.doi.org/10.1016/j. celrep.2014.03.008 (2014) 